\title{
GAIA Level 3 Neonatal Encephalopathy
}

National Cancer Institute

\section{Source}

National Cancer Institute. GAIA Level 3 Neonatal Encephalopathy. NCI Thesaurus. Code C128731.

GAIA Level 3 Neonatal Encephalopathy is defined by three criteria: first, a newborn infant (1-28 days of life) born at or beyond 35 weeks of gestation; second, an abnormal level of alertness or seizures; third, none of the following: a) difficulty with initiating or maintaining respiration; b) depression of muscle tone. 